\title{
The HECTD3 E3 ubiquitin ligase facilitates cancer cell survival by promoting K63-linked polyubiquitination of caspase-8
}

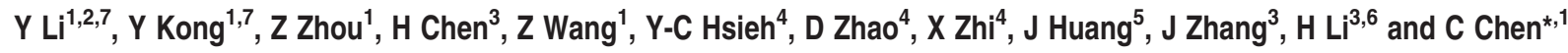

Apoptosis resistance is a hurdle for cancer treatment. HECTD3, a new E3 ubiquitin ligase, interacts with caspase-8 death effector domains and ubiquitinates caspase-8 with K63-linked polyubiquitin chains that do not target caspase-8 for degradation but decrease the caspase-8 activation. HECTD3 depletion can sensitize cancer cells to extrinsic apoptotic stimuli. In addition, HECTD3 inhibits TNF-related apoptosis-inducing ligand (TRAIL)-induced caspase-8 cleavage in an E3 ligase activity-dependent manner. Mutation of the caspase-8 ubiquitination site at K215 abolishes the HECTD3 protection from TRAIL-induced cleavage. Finally, HECTD3 is frequently overexpressed in breast carcinomas. These findings suggest that caspase-8 ubiquitination by HECTD3 confers cancer cell survival.

Cell Death and Disease (2013) 4, e935; doi:10.1038/cddis.2013.464; published online 28 November 2013

Subject Category: Cancer

Apoptosis is a critical cellular process required for the maintenance of tissue homeostasis; dysregulation of apoptosis is a major contributor to human pathologies including cancer. Caspases, a family of cysteine proteases, are common death effector molecules that cleave a number of different substrates. ${ }^{1}$ Caspase activation can be initiated by the mitochondrial (intrinsic) or death receptor (extrinsic) pathways. ${ }^{2}$ The extrinsic pathway is activated by TNF family ligands, including TNF $\alpha$, FasL, and the TNF-related apoptosis-inducing ligand (TRAIL) through the respective binding with their specific receptors. Subsequently, an initial caspase, pro-caspase-8, is recruited to the oligomeric membraneassociated death-inducing signaling complex (DISC) through FADD, resulting in pro-caspase-8 oligomerization and self-cleavage. ${ }^{3,4}$ Activated caspase- 8 induces apoptosis by directly cleaving effector caspases, e.g., caspase-3 and $-7 .{ }^{5}$ The available evidence suggests that caspase activation is regulated by ubiquitination. Several inhibitors of apoptosis (IAP) E3 ligases, XIAP and cIAP1/2, inhibit caspase-9, -7, and -3 activation through direct interaction ${ }^{6-8}$ and ubiquitination. ${ }^{9,10}$ Caspase-8 ubiquitination at its C-terminus by a CUL3-based E3 ligase complex promotes caspase-8 activation and apoptosis. ${ }^{11}$ Peng et al. reported that EGF induces caspase-8 phosphorylation, ubiquitination, and degradation, although the responsible E3 ligase is unknown. ${ }^{12}$ E3 ubiquitin ligases $\mathrm{SIAH} 2$ and $\mathrm{POSH}$ have been shown to inhibit caspase-8 activity; ${ }^{13}$ however, whether these E3 ligases ubiquitinates caspase-8 has not been tested. TRAF2-mediated K48-linked polyubiquitination on the large catalytic domain ( $p 18$ ) of caspase-8 increases the degradation of active caspase- 8 and the signal threshold for death receptor-mediated apoptosis. ${ }^{14}$ Consistently, inhibition of the proteasomal degradation of p18 sensitizes cancer cells to TRAIL-induced apoptosis. ${ }^{15,16}$

Ubiquitination regulates multiple cellular processes including apoptosis. The ubiquitin (Ub) can be conjugated to the substrate's lysine $(K)$ residues through isopeptide bonds. Protein ubiquitination is sequentially mediated by three enzymes: the ubiquitin-activating enzyme (E1), ubiquitinconjugating enzyme (E2), and ubiquitin ligase (E3) that controls substrate specificity. Ub is conjugated either as a single moiety or as polyubiquitin chains linked through K48, $\mathrm{K} 63$, or other $\mathrm{K}$ residues of $\mathrm{Ub}$ with different functional consequences. K48-linked polyubiquitin chains target substrates to the $26 \mathrm{~S}$ proteasome for degradation while K63-linked polyubiquitin chains initiate non-degradation signaling. ${ }^{17}$

E3 ligases partition into two subfamilies; the RING finger domain-containing E3s and the HECT (homologous to E6-AP

\footnotetext{
${ }^{1}$ Key Laboratory of Animal Models and Human Disease Mechanisms of Chinese Academy of Sciences \& Yunnan Province, Kunming Institute of Zoology, Kunming, Yunnan 650223, China; ${ }^{2}$ State Key Laboratory of Molecular Oncology, Cancer Institute, Chinese Academy of Medical Sciences and Peking Union Medical College, Beijing 100021, China; ${ }^{3}$ Wadsworth Center, New York State Department of Health, 120 New Scotland Ave, Albany, NY 12208, USA; ${ }^{4}$ The Center for Cell Biology and Cancer Research, Albany Medical College, 47 New Scotland Ave, Albany, NY 12208, USA; ${ }^{5}$ Department of Pathology, Medical College of Wisconsin, Milwaukee, WI 53226, USA and ${ }^{6}$ Department of Biomedical Sciences, School of Public Health, University at Albany, State University of New York, PO Box 509, Albany, NY 12201, USA ${ }^{*}$ Corresponding author: C Chen, Key Laboratory of Animal Models and Human Disease Mechanisms of Chinese Academy of Sciences \& Yunnan, Kunming Institute of Zoology, 32 Jiaochang Dong Rd, Kunming 650223, China. Tel: +86 871 5181944; Fax: +86 871 5181945; E-mail: chenc@mail.kiz.ac.cn

${ }^{7}$ These authors contributed equally to this work.

Keywords: ubiquitination; apoptosis; breast cancer; HECTD3; caspase-8

Abbreviations: APC, anaphase-promoting complex; C, cysteine; DED, death effector domains; DISC, death-inducing signaling complex; DOC, destruction of cyclin; HECT, homologous to E6-AP COOH terminus; IAP, inhibitor of apoptosis; IHC, immunohistochemical analysis; IP, immunoprecipitation; K, lysine; TRAIL, TNF-related apoptosis-inducing ligand; Ub, ubiquitin; WB, western blotting; WT, wild type

Received 24.6.13; revised 07.10.13; accepted 23.10.13; Edited by GM Fimia
} 
$\mathrm{COOH}$ terminus) domain-containing E3s. ${ }^{18,19}$ All 28 HECTtype E3s contain a conserved C-terminal HECT domain and a highly variable $\mathrm{N}$-terminal domain that is responsible for substrate binding. ${ }^{20-24}$ The HECT domain-containing 3 (HECTD3) E3 ligase contains an $\mathrm{N}$-terminal DOC (destruction of cyclin) domain. The DOC domain has been linked to substrate recognition in several E3 ligases including the anaphase-promoting complex subunit 10 (APC10/DOC1), ${ }^{25}$ PARC, CUL7, and HERC2. ${ }^{26} \mathrm{~N}$-terminal-truncated HECTD3 targets Tara (Trio-associated repeat on actin) for ubiquitinmediated degradation. ${ }^{27}$ In addition, HECTD3 depletion induces multipolar spindle formation in $\mathrm{HeLa}$ cells. ${ }^{27}$ Moreover, HECTD3 has been shown to ubiquitinate Syntaxin-8. ${ }^{28}$ Most recently, we reported that HECTD3 ubiquitinates MALT1 with nondegradative polyubiquitin chains, stabilizes MALT1, and confers cancer cells to cisplatin. ${ }^{29}$ The role and action mechanism of HECTD3 in cancer, however, is not completely understood.

\section{Results}

HECTD3 interacts with caspase-8 through the DOC/DED domains. HECTD3 ubiquitin E3 ligase interacts with MALT1, ${ }^{29}$ which has been reported to form complex with Caspase-8. ${ }^{30}$ We wondered whether HECTD3 interacts with caspase-8. The protein interaction between HECTD3 and caspase-8 was confirmed by co-immunoprecipitation (IP). HECTD3 specifically interacted with the endogenous caspase-8 but not caspase-3 and -7 compared with HECTD3 1 1-511, which does not have the DOC domain (Figures 1a and b). The HECTD3-caspase-8 protein interaction was further confirmed by a reciprocal co-immunoprecipitation experiment (Figure 1c). The GST pull-down experiment indicated that the purified recombinant HECTD3 protein from E.coli (Supplementary Figure S1A) interacted with the caspase- 8 protein translated in vitro using a cell-free in vitro translation system (Figure 1d). In contrast, HECTD3 failed to pull-down the in vitro translated caspase-3 protein (Figure 1d). These results indicated that HECTD3 specifically and directly interacts with caspase-8. We further demonstrated that the endogenous HECTD3 protein interacted with the endogenous caspase-8 proteins in $\mathrm{HeLa}$ (Figure 1e). These results suggest that HECTD3 and caspase-8 interact with each other at the physiological level. The localization of Flag-HECTD3 and caspsase-8 in HEK293T cells were evaluated by immunofluorescence staining. As shown in Figure 1f, both Flag-HECTD3 and caspsase-8 are predominately localized in the cytoplasm.

The HECTD3 protein domain responsible for caspase-8 binding was mapped. As shown in Figure 1g, depletion of the $\mathrm{N}$-terminal 511 residues from HECTD3 resulted in the loss of binding with caspase-8. The smallest HECTD3 domain responsible for caspase-8 binding was mapped to a region between 109 and 393 that harbors the DOC domain (Figure 1a). Compared with the wild-type (WT) HECTD3, several HECTD3 deletion mutants, namely $\Delta 1-215$, $\Delta 216-393, \Delta 394-511$, and $\Delta 512-861$, displayed enhanced binding to caspase-8 (Supplementary Figure S1B). Finally, GST pull-down assays indicated that $\mathrm{H} 109-393$ was sufficient to bind to caspase-8 (Figure 1h). Thus, the DOC domain
(216-393) alone was insufficient to bind to caspase-8, and the region between 109 and 393 from HECTD3 was necessary and sufficient for caspase-8 binding.

Caspase-8 contains two death effector domains (DEDs) and two caspase domains (p18 and p10) (Figure 1a). DEDs are responsible for the caspase-8 recruitment into DISC through FADD. To map the caspase-8 domain responsible for the association with HECTD3, we expressed the DED and caspase domains of caspase-8 as GST-fusion proteins in HEK293T and performed the GST pull-down assay. Our experimental results indicated that either DED1 or DED2 is sufficient to interact with HECTD3 by performing the GST pull-down assay (Figure $1 \mathrm{i}$ and Supplementary Figure S1C). Another DED domain-containing protein FLIP does not interact with HECTD3 (Supplementary Figure S1D).

HECTD3 ubiquitinates caspase-8 with K63-linked polyubiquitin chains. As shown in Figure 2a, HECTD3 significantly increased caspase-8 polyubiquitination compared with the vector control. It is noted that HECTD3 does not affect the steady-state level of pro-caspase-8 in HEK293T cells, indicating that overexpression of HECTD3 does not target pro-caspase-8 for degradation (Figure 2a). It is possible that HECTD3 modifies caspase-8 with nonK48-linked polyubiquitin chains. The linkage of caspase-8 polyubiquitin chains mediated by HECTD3 was analyzed by using linkage-specific antibodies that specifically recognize K48- or K63-linked polyubiquitin chains under denaturing conditions. ${ }^{31}$ HECTD3-mediated caspase-8 ubiquitination could be detected by the antibody against K63-linked polyubiquitin chains, but not by the antibody against K48-linked polyubiquitin chains (Figure 2b). To further confirm this result, different ubiquitin mutants including K48-only, K63-only, K48R, K63R, and K0 were used. Consistently, HECTD3-mediated caspase-8 polyubiquitination was supported by WT, K63-only, or K48R Ub, but not by K48-only, K63R, or K0 Ub (Figure 2c). These results clearly indicate that HECTD3 ubiquitinates caspase-8 with K63linked polyubiquitin chains. Finally, we demonstrated that endogenous ubiquitination of caspase-8 is decreased when endogenous HECTD3 is knocked down in MDA-MB-231 (Supplementary Figure S2E). In contrast to the CUL3-based E3 ligase, ${ }^{11}$ HECTD3-mediated caspase-8 ubiquitination was not induced by TRAIL (Supplementary Figure S2F).

The catalytic cysteine residue of HECTD3 for caspase-8 is C823. Almost all HECT-type E3 ligases contain a C-terminal catalytic HECT domain with a conserved catalytic cysteine (Cys) located in 'HTCFN' box ${ }^{32}$ (Figure 2d). The catalytic Cys residue is conserved in the HECTD3, although HECTD3 does not have such a box. When HECTD3 C823 was mutated into $A$, the E3 ligase activity was completely abolished as determined by performing the in vitro HECTD3 self-ubiquitination assay (Figure $2 \mathrm{e}$ ). Consistently, ubiquitination of caspase-8 by HECTD3 was decreased by the C823A mutation in HEK293T cells (Figure 2f). As HECTD3C823A appeared to still increase the ubiquitination of caspase-8 compared with the vector control (Figure 2f), we tested whether other C-residues contribute to the caspase-8 
a

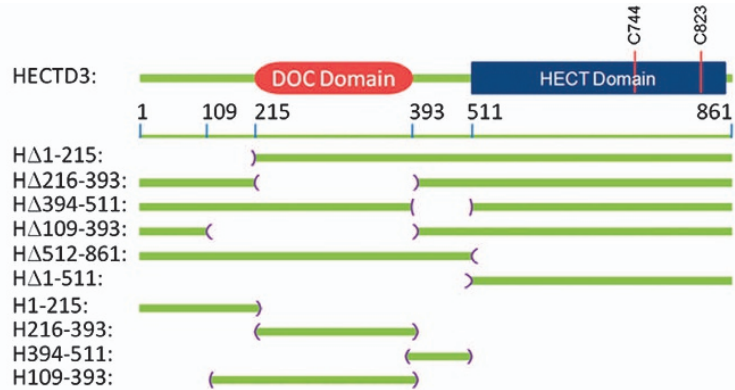

Caspase-8:
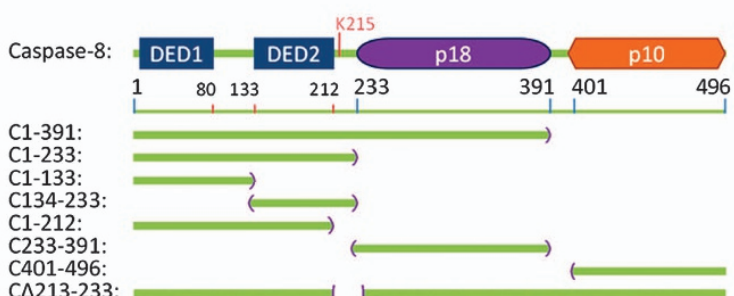

C $\triangle 213-233$

d

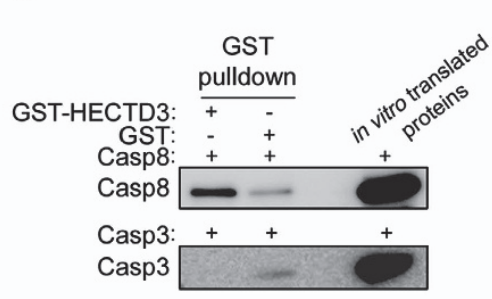

g

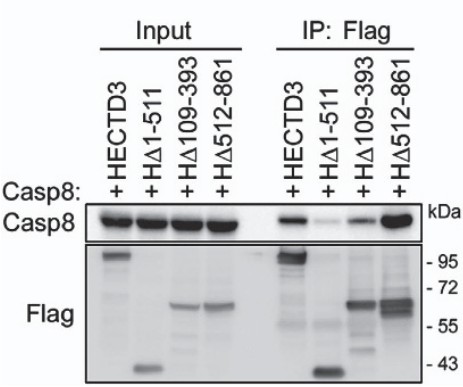

h

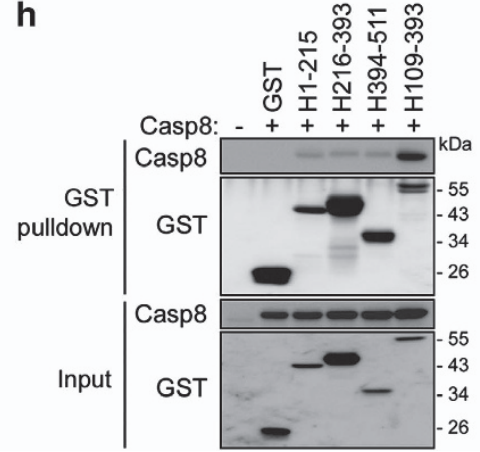

e

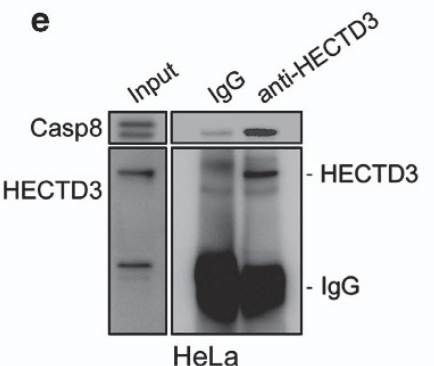

b

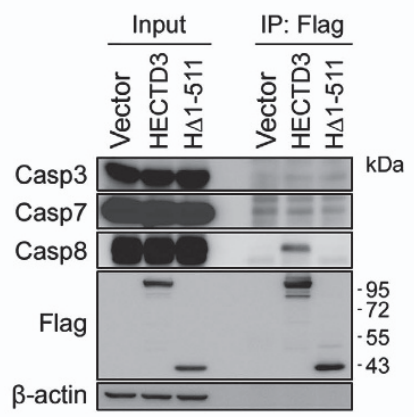

C
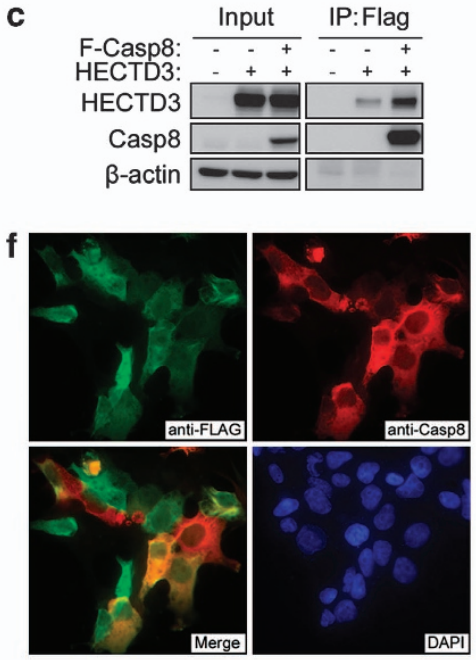

i

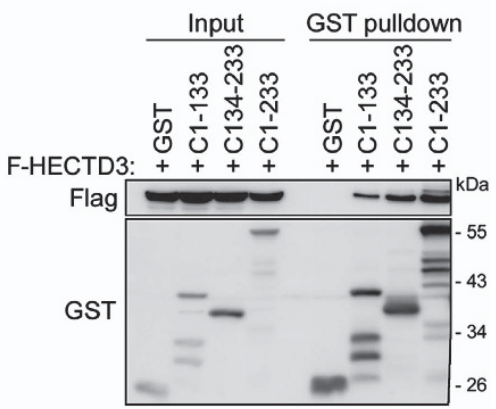

Figure 1 HECTD3 interacts with caspase-8 through the DOC and DED domains. (a) Schematic representation of the HECTD3 and caspase-8 proteins and their mutants. (b) WT HECTD3 interacts with endogenous caspase-8, but not caspase-3 and -7. Flag-HECTD3, Flag-H $\Delta 1-511$ and an empty vector were transfected into HEK293T for 2 days. Immunoprecipitation was performed using the anti-FLAG M2 beads. (c) Caspase-8 interacts with HECTD3. Flag-caspase-8 or an empty vector was co-transfected with HECTD3 into HEK293T for 2 days. Immunoprecipitation was performed using the anti-FLAG M2 beads. (d) HECTD3 directly binds to caspase-8 but not caspase-3 in vitro. The recombinant GST-HECTD3 and GST were purified from E.coli (Supplementary Figure S1A) and were incubated with in vitro-translated caspase-8 and caspase-3 proteins, respectively, with Glutathione-Sepharose $4 \mathrm{~B}$ slurry beads overnight at $4{ }^{\circ} \mathrm{C}$. The beads were washed three times with $1 \mathrm{ml}$ of $1 \times$ cell lysis buffer. The proteins were resuspended with 20-50 $\mu \mathrm{l}$ of SDS sample buffer and analyzed by WB. (e) The endogenous HECTD3 protein forms a complex with the endogenous caspase-8 protein in HeLa. The endogenous HECTD3 was immunoprecipitated by the anti-HECTD3 Ab. Rabbit lgG was used as a negative control. (f) Both HECTD3 and caspase-8 are predominantly localized in the cytoplasm. The localization of Flag-HECTD3 and caspase-8 proteins was examined by immunofluorescence staining in HEK293T cells after co-transfection. DAPI was used to stain cell nuclei. (g) $\mathrm{H} \Delta 1-511$ and $\mathrm{H} \Delta 109-393$ significantly decrease the binding affinity with caspase-8. Flag-HECTD3 and its mutants were co-transfected with caspase-8 into HEK293T for 2 days. Immunoprecipitation was performed using the anti-FLAG M2 beads. (h) GST-fused H109-393 is sufficient to bind to caspase-8 as determined by performing the GST pull-down assay. (i) Flag-HECTD3 binds to both DED1 and DED2 of caspase-8 as determined by the GST pull-down assay

ubiquitination. Six other C-residues in the HECT domain were mutated to $\mathrm{A}$, and their E3 ligase activities toward caspase-8 in HEK293T were assessed. Compared with WT and other mutants, the $\mathrm{C} 744 \mathrm{~A}$ and $\mathrm{C} 744,823 \mathrm{~A}$ double mutants efficiently decreased caspase-8 polyubiquitination by HECTD3 (Figure $2 f$ and Supplementary Figure S2A). C744 is conserved in several other HECT-type E3 ligases (Figure $2 d$ ). Mutation of five other $C$ residues into $A$ in the 
a

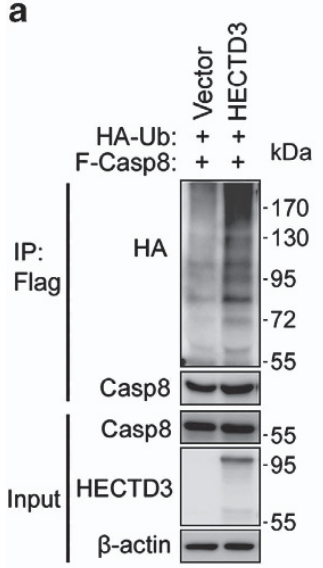

b

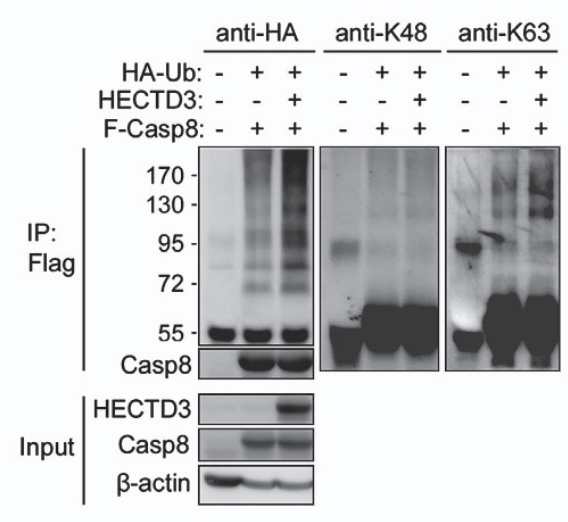

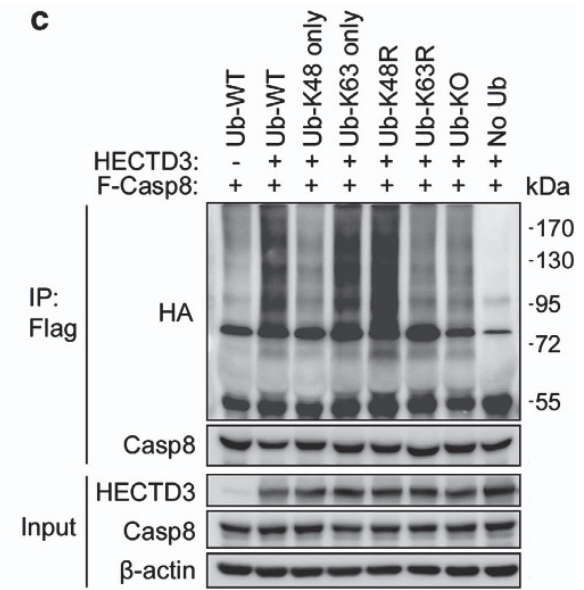

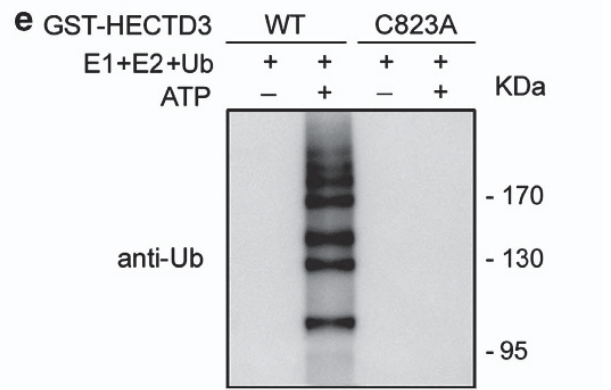

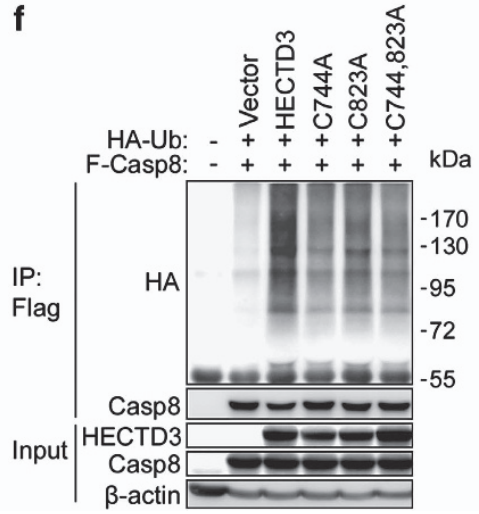

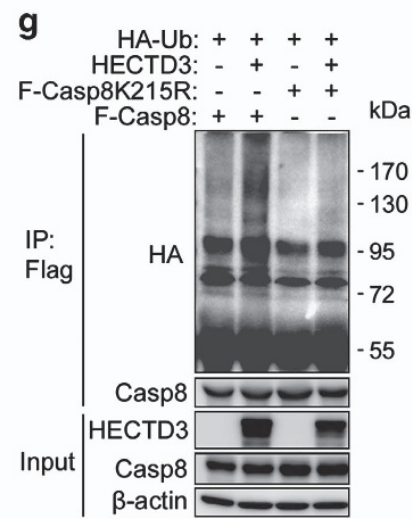

Figure 2 HECTD3 ubiquitinates caspase-8 with K63-linked polyubiquitin chains at K215. (a) HECTD3 ubiquitinates caspase-8. HEK293T cells were co-transfected with expressing plasmids for HA-Ub, HECTD3, and Flag-caspase-8 as indicated for 2 days. The ubiquitinated Flag-caspase-8 proteins were detected by an anti-HA antibody after immunoprecipitation caspase-8 by anti-Flag M2 beads under denaturing conditions. (b) The ubiquitination of caspase-8 by HECTD3 was assessed with linkage-specific antibodies that recognize K48- or K63-linked polyubiquitin chains. (c) Different HA-Ub mutants were used to assess the HECTD3-mediated caspase-8 polyubiquitin chain linkage in HEK293T. (d) Alignment of the HECT domains of 10 HECT type E3 ligases using the Glustal W multiple alignment program. ${ }^{47}$ HECTD3 does not have the conserved 'HTCFN' catalytic loop. (e) HECTD3-C823A loses the E3 ubiquitin ligase activity, as determined by the in vitro ATP-dependent ubiquitination assay. UbcH5b was used as the E2, which can mediate the K63-linked polyubiquitination. ${ }^{43}$ (f) Both $\mathrm{C} 823$ and $\mathrm{C744}$ contribute to the E3 ligase activity of HECTD3 toward caspase-8. In contrast to WT HECTD3, the HECTD3-C744A, -C823A, and -C744, 823A mutants cannot efficiently ubiquitinate caspase-8 in HEK293T. (g) In contrast to WT caspase-8, the caspase-8K215R mutant cannot be efficiently ubiquitinated by HECTD3 in HEK293T

HECT domain did not affect the polyubiquitination of caspase-8 by HECTD3 (Supplementary Figure S2A).

HECTD3 ubiquitinates pro-caspase-8 at K215. To identify which lysine $(\mathrm{K})$ residue of caspase-8 is ubiquitinated, different caspase-8 mutants with DEDs (these mutants can interact with HECTD3) were evaluated for HECTD3mediated ubiquitination. HECTD3 ubiquitinated WT caspase-8, C1-391, but not C1-212 (Supplementary Figure S2B), implying that the HECTD3-mediated caspase-8 ubiquitination sites may be located between 213 and 391. HECTD3 did not efficiently ubiquitinate caspase8 $\Delta 213-233$ (Supplementary Figure S2C). There is only one $\mathrm{K}$ (215) residue in this region; therefore, the caspase-8K215R mutant was generated. HECTD3 indeed did not ubiquitinate this mutant (Figure 2g), although the K215R 
mutation did not affect the interaction between caspase-8 and HECTD3 (Supplementary Figure S2D).

It is well known that caspase-8 cleavage occurs at D384 followed by D210 and D216. ${ }^{33,34}$ To test whether caspase-8 cleavage has a role in HECTD3-mediated caspase-8 polyubiquitination, we measured the ubiquitination of an inactive caspase-8 mutant $\mathrm{C} 377 \mathrm{~A}$ that prevents caspase-8 selfcleavage events. ${ }^{35}$ As shown in Supplementary Figure S3A, the cleaved caspase-8 band is detected when WT caspase-8 is overexpressed; however, it is undetectable when caspase-8 C377A is overexpressed. Consistently, the basal level of ubiquitination for caspase-8(C377A) is higher than WT caspase-8. Nevertheless, HECTD3 increased the ubiquitination of both WT caspase-8 and caspase-8 (C377A) mutant, suggesting that HECTD3 ubiquitinates pro-caspase-8 independent of its cleavage.

Depletion of HECTD3 increases caspase-8 activation and apoptosis induced by the extrinsic apoptosis pathway. To test if HECTD3 regulates the caspase-8 activation through ubiquitinating caspase-8, HECTD3 was stably knocked down by shRNA in the HeLa cervical cancer cell line (Figure 3a) and MDA-MB-231 breast cancer cell line (Figure $3 b$ ). As caspase-8 is mainly activated by the extrinsic apoptotic pathway, stable HECTD3 knockdown cells were treated with different dosages of TRAIL. HECTD3 depletion promotes the caspase- 8 cleavage (Figures $3 a$ and b) and activity (Supplementary Figure S3B) in the presence of TRAIL in a dose-dependent manner. In addition, HECTD3depleted cells showed increased apoptosis as indicated by the increase of cleaved caspase-3 and PARP in the presence of TRAIL (Figure 3a). Similar results were observed in both HeLa and MDA-MB-231 treated with the Fas antibody and TNF $\alpha$ (Figures $3 a$ and b). Transient knockdown of HECTD3 by two different siRNAs also sensitized HeLa cells to TRAIL-induced caspase-8 activation and apoptosis (Supplementary Figure S4A).

The enhancement of TRAIL-induced apoptosis by HECTD3 depletion was further assessed by the Annexin V/7-AAD staining, the cell viability assay, and the clonogenic assay. As shown in Figure 3c, HECTD3 knockdown in MDA-MB-231 significantly increased the percentage of Annexin V-positive apoptotic cells. In addition, the cell viability of MDA-MB-231 HECTD3sh cells was significantly less than that of Lush cells after the TRAIL treatment (Figure 3d). The clonogenic assay showed that HECTD3 knockdown markedly decreased the colony formation in the presence of TRAIL (Figure 3e).

HECTD3 decreases the caspase- 8 recruitment and inhibits its activation. To investigate the potential mechanism by which HECTD3 inhibits caspase-8 activation, we examined TRAIL-induced caspase- 8 recruitment into DISC. As shown in Figure 4a, more endogenous caspase-8 proteins were recruited into DISC when endogenous HECTD3 was knocked down in MDA-MB-231. HECTD3 itself was not recruited into DISC (Figure 4a).

To test whether HECTD3 inhibits caspase-8 cleavage and activity by ubiquitinating caspase-8, TRAIL-mediated caspase-8 cleavage and activity in HEK293T was measured. WT HECTD3 significantly inhibited TRAIL-induced caspase-8 cleavage (Figure 4b, lane 5 compared with lane 4) and activity (Supplementary Figure S3C); however, the catalytic inactive HECTD3m (lane 6) did not inhibit TRAIL-induced caspase-8 cleavage and activity (Supplementary Figure S3C). Compared with that of WT caspase-8 (Figure 4b, lane 5), the cleavage of caspase-8-K215R induced by TRAIL (Figure 4b, lane 11) could not be blocked by HECTD3 overexpression. Consistently, the caspase-8 activity is neither reduced by HECTD3 (Supplementary Figure S3C). TRAF2 has been shown to target caspase-8 p18 for proteasomal degradation. ${ }^{14}$ The proteasome inhibitor MG132 only slightly increased the p18 protein level in MDA-MB-231 cells treated with TRAIL (Supplementary Figure S3D), suggesting that HECTD3 does not target p18 for proteasomal degradation.

HECTD3 overexpression was evaluated as a potential rescue of the HECTD3 knockdown-induced caspase-8 cleavage and apoptosis increase in the presence of TRAIL. HECTD3 shRNA-resistant WT HECTD3 ${ }^{R}$ and HECTD3m ${ }^{R}$ were stably expressed in the HECTD3-depleted MDA-MB231 cells. As shown in Figures $4 c$ and $d$, the expression of HECTD ${ }^{R}$ protected MDA-MB-231 cells from TRAIL-induced caspase- 8 cleavage and cell viability decrease; however, the catalytic inactivated HECTD3m ${ }^{R}$ did not. These results not only suggest that the pro-apoptosis phenotype caused by HECTD3 shRNA is not an off-target effect but also further confirm that the E3 ligase activity of HECTD3 is required to suppress TRAIL-induced apoptosis.

HECTD3 promotes cell survival through caspase-8. Previously, we demonstrated that HECTD3 promotes cancer cell survival partially through MALT1. ${ }^{29}$ To test if HECTD3 decreases TRAIL-induced apoptosis through caspase-8, a rescue experiment was performed by depleting caspase- 8 and HECTD3 together using siRNA in both MDA-MB-231 and HeLa cells followed by treatment of the cells with TRAIL. Caspase-8 ablation almost completely abrogates HECTD3 siRNA-induced apoptosis increase in the presence of TRAIL, as determined by measuring caspase-8, -3, -7, and PARP cleavage as well as cell viability (Supplementary Figure S4B-E). In contrast, caspase- 9 ablation has no significant effect on HECTD3 siRNA-induced apoptosis increase in the presence of TRAIL (Supplementary Figure S4F).

HECTD3 is frequently overexpressed in breast cancers. HECTD3 mRNA is strongly expressed in the human salivary gland, liver, thyroid, kidney, muscle, and brain, but weakly expressed in the ovary, prostate, skin, cervix, and mammary gland (Supplementary Figure S5A). The HECTD3 expression in different human tissues was further validated by immunohistochemical analysis (IHC) (Supplementary Figure S5B-C and data not shown).

As HECTD3 mRNA is weakly expressed in normal mammary glands, the HECTD3 mRNA levels were measured by qRT-PCR in 30 breast cancer cell lines. Compared with four nontransformed breast cell lines (HMEC, 184A1, MCF10A, and 184B5), 19 of 30 (63.3\%) of breast cancer cell lines overexpress HECTD3 by at least twofold (Figure 5a).

To further validate the GRT-PCR results, northern blot analyses for HECTD3 were performed in several breast cancer cell lines. As expected, a single $\sim 3.6-\mathrm{Kb}$ mRNA band 
a

\begin{tabular}{|c|c|c|c|c|c|c|c|c|c|c|c|c|c|c|c|c|}
\hline \multicolumn{4}{|c|}{ TRAIL } & \multicolumn{7}{|c|}{ Fas $A b$} & \multicolumn{5}{|c|}{$\mathrm{TNF} \alpha$} & \multirow[b]{2}{*}{$\mathrm{ng} / \mathrm{ml}$} \\
\hline 0 & 2 & & 50 & 0 & & 100 & & 200 & & & & .5 & 5 & 5 & 10 & \\
\hline$\stackrel{\frac{c}{\omega}}{\stackrel{5}{口}}$ & 兵 & $\frac{c}{\frac{0}{1}}$ & $\stackrel{\frac{c}{3}}{\frac{c}{1}}$ & 臬 & $\frac{c}{10}$ & ב & $\frac{c}{\frac{0}{1}}$ & $\stackrel{\frac{c}{\omega}}{\frac{1}{3}}$ & & $\frac{\frac{c}{\infty}}{1}$ & 䍃 & $\frac{c}{\frac{0}{1}}$ & 兵 & $\frac{c}{\frac{c}{1}}$ & 皇 $\frac{c}{\text { क }}$ & $\mathrm{KDa}$ \\
\hline
\end{tabular}

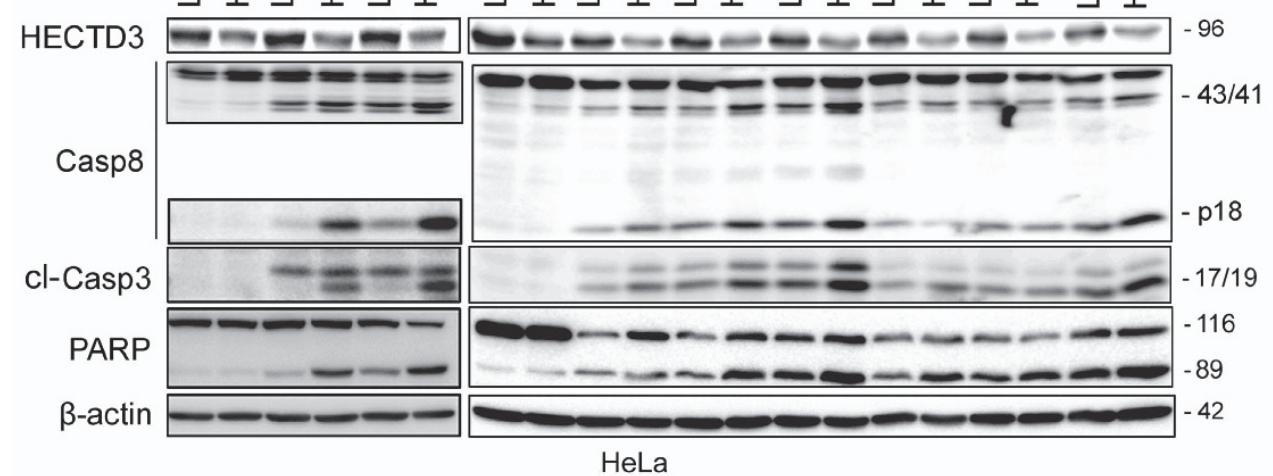

b

\begin{tabular}{|c|c|c|c|c|c|c|c|c|c|}
\hline & TRAIL & & & & & $\mathrm{Ab}$ & & \multicolumn{2}{|c|}{$\mathrm{TNF} \alpha$} \\
\hline 0 & 12.5 & 25 & 50 & 0 & 100 & 200 & 400 & 2.5 & 5 \\
\hline 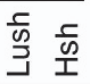 & 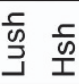 & $\stackrel{\frac{c}{\omega}}{\frac{c}{\omega}}$ & 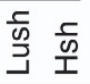 & 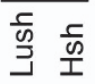 & $\stackrel{\frac{c}{\omega}}{\frac{c}{3}}$ & 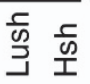 & $\stackrel{\text { क }}{\stackrel{5}{口}}$ & 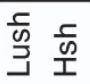 & 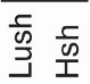 \\
\hline
\end{tabular}
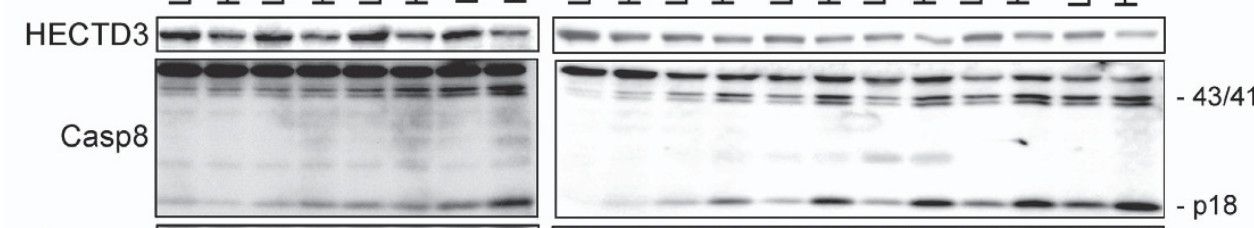

cl-Casp3
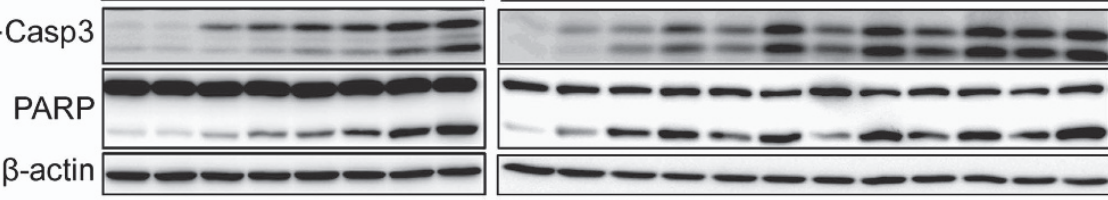

MDA-MB-231

C
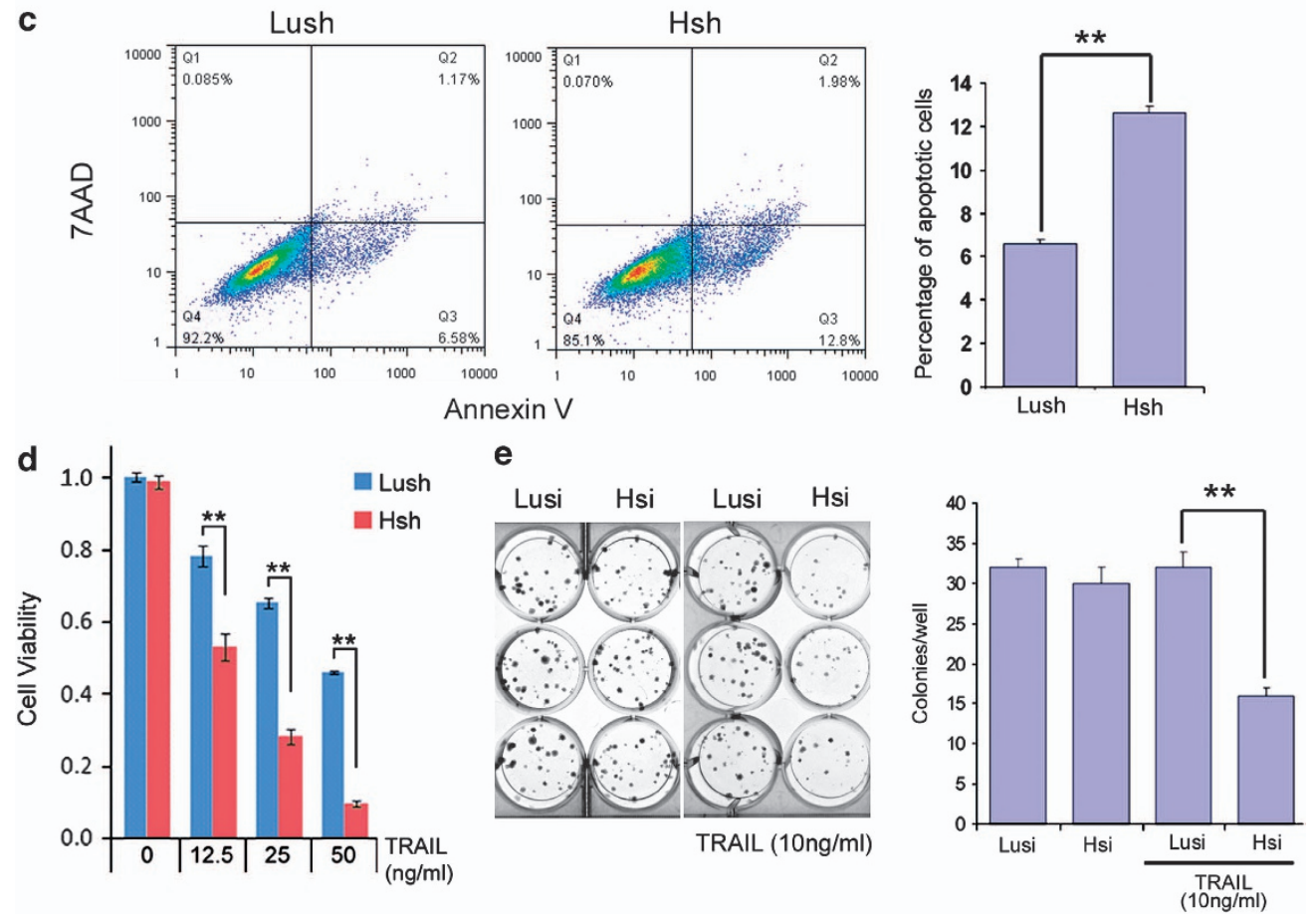
was detected in all samples examined (Supplementary Figure S6A). The HECTD3 mRNA levels are higher in cancer cell lines than those in immortalized cell lines (Supplementary Figure S6A). To test if the HECTD3 gene undergoes genetic alterations in breast cancer, the DNA copy-number of HECTD3 in 31 breast cancer cell lines was evaluated. Compared with the genomic DNA from normal human cells and three immortalized cell lines (MCF10A, 184A1, and 184B5), 15 of the 31 breast cancer cell lines (48.4\%) showed gains in gene copy-number for HECTD3 (Supplementary Figure S6B). There is a significant correlation between gain of the HECTD3 gene copy-number and the HECTD3 mRNA overexpression in breast cancer cell lines (Supplementary Table S1).

The endogenous HECTD3 protein levels in breast cancer cell lines were measured by western blotting (WB). The HECTD3 protein band was detected at $96 \mathrm{KDa}$ in all samples (Figure 5b). In agreement with the mRNA expression
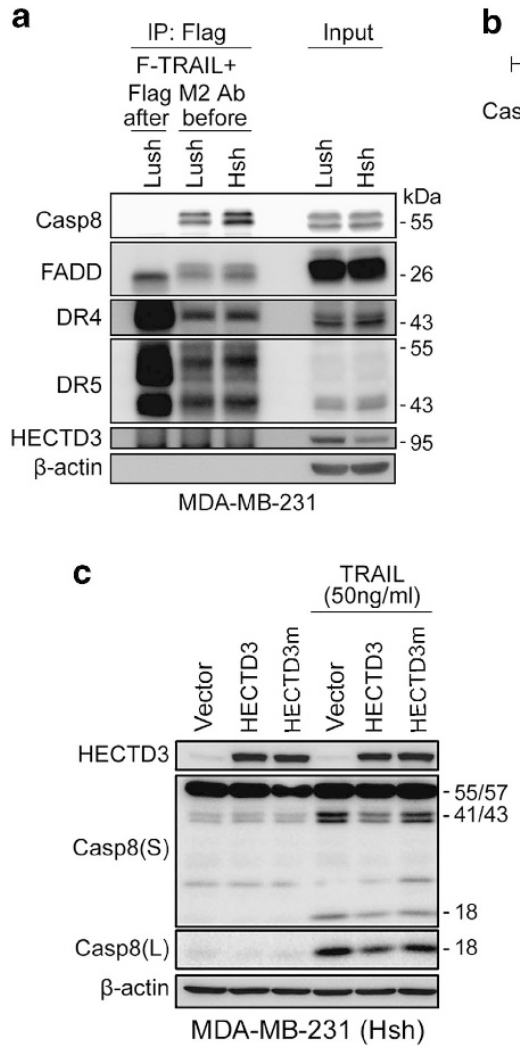
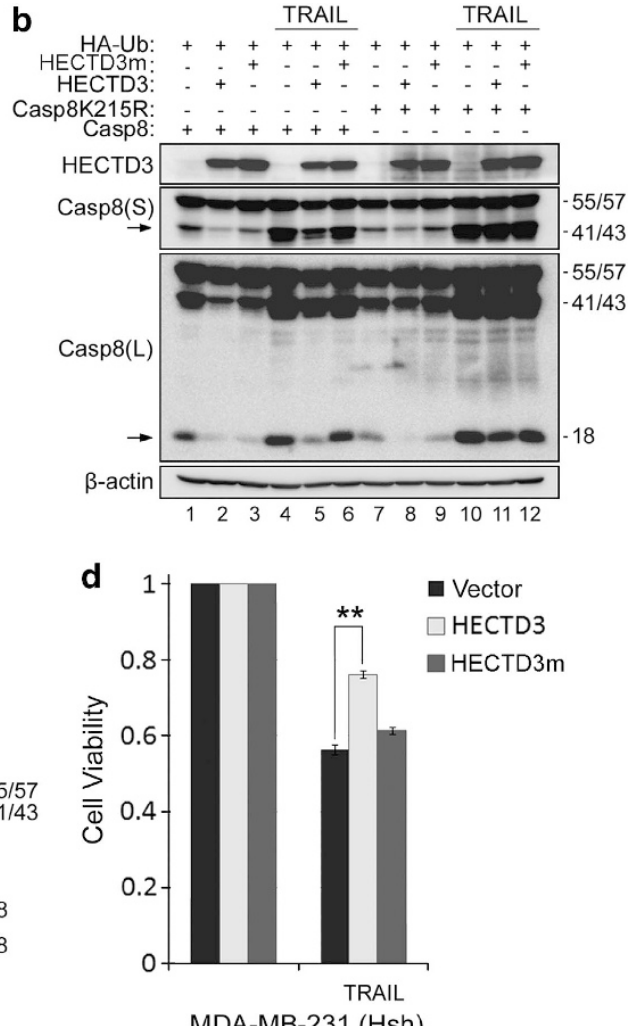

Figure 4 HECTD3 decreases the caspase-8 recruitment and inhibits its activation. (a) HECTD3 ablation increases the caspase-8 association with DISC in MDA-MB-231 cells treated with Flag-TRAIL. Stable HECTD3 knockdown and control MDA-MB-231 cells were treated with Flag-TRAIL for 30 min before or after collection. DISC formation was examined by immunoprecipitation using anti-Flag M2 beads and WB. (b) HECTD3 inhibits TRAIL-induced caspase-8 cleavage (41/43 and 18 KDa cleaved caspase-8 bands are indicated by arrows) in HEK293T in HECTD3 E3 ligase activity and caspase-8 ubiquitination site-dependent manners. HEK293T cells were transfected with indicated constructs overnight and treated with $50 \mathrm{ng} / \mathrm{ml}$ TRAIL for $4 \mathrm{~h}$. HECTD3m (C744, 823A) cannot decrease TRAIL-induced caspase-8 cleavage as WT HECTD3 (lane 6 versus 5). In addition, HECTD3 cannot decrease TRAIL-induced cleavage of caspase-8-K215R (lane 11 versus 5). L, long exposure; S, short exposure. (c) Re-expression of shRNA resistant HECTD3 ${ }^{R}$ in endogenous HECTD3-depleted MDA-MB-231 cells decreases TRAIL-induced cleavage of caspase-8 in an E3 ligase activity-dependent manner. Endogenous HECTD3 was silenced by shRNA. Following that, HECTD3 ${ }^{R}$ and HECTD3m $(C 744,823 A)^{R}$ were stably introduced by lentiviruses.(d) HECTD3 ${ }^{R}$ significantly decreases TRAIL $(50 \mathrm{ng} / \mathrm{ml})$-induced loss of cell viability in an E3 ligase activity-dependent manner in MDA-MB-231. Cell viability was measured by performing the SRB assay. Error bars are S.D. from three independent experiments. ${ }^{* *} P<0.01$ (t-test)

Figure 3 HECTD3 depletion resulted in increase of caspase-8 activation and apoptosis induced by TRAIL, Fas Ab, and TNF $\alpha$ (a) HECTD3 depletion in HeLa by shRNA led to increase of caspase-8, -3, and PARP cleavage (cl-) after treatment with different dosages of TRAlL (25-50 ng/ml), anti-CD95 Ab (100-400 ng/ml with $10 \mu \mathrm{g} / \mathrm{ml}$ cycloheximide (CHX, blocking NF- $\kappa$ B pro-survival target gene expression)), and TNF $\alpha(2.5-10 \mathrm{ng} / \mathrm{ml}$ with $10 \mu \mathrm{g} / \mathrm{ml} \mathrm{CHX)}$ for $16 \mathrm{~h}$. Lush means control shRNA against the luciferase gene. Hsh means shRNA against HECTD3. (b) HECTD3 depletion in MDA-MB-231 by shRNA resulted in increase of caspase-8, -3 , and PARP cleavage after treatment with different dosages of TRAlL (25-50 ng/ml), anti-CD95 Ab (100-400 ng/ml with $10 \mu \mathrm{g} / \mathrm{ml} \mathrm{CHX),} \mathrm{and} \mathrm{TNF} \alpha(2.5-5 \mathrm{ng} / \mathrm{ml}$ with $10 \mu \mathrm{g} / \mathrm{ml} \mathrm{CHX)} \mathrm{for} 16 \mathrm{~h}$. (c) HECTD3 depletion in MDA-MB-231 significantly increased Annexin V-positive apoptotic cells. Stable cells were treated with $50 \mathrm{ng} / \mathrm{ml}$ TRAIL for $16 \mathrm{~h}$ and stained with FITC-Annexin V and 7-AAD. The stained cells were analyzed by flow cytometry. The quantitative results are shown on the right panel. Error bars are S.D. from three independent experiments. ${ }^{* *} P<0.01$, t-test. (d) HECTD3 depletion decreased MDA-MB-231 cell viability in the presence of different dosages of TRAIL for 2 days, as determined by the SRB assay. Error bars are S.D. from three independent experiments. ${ }^{* \star} P<0.01$, $t$-test. Lusi means control siRNA against the luciferase gene. Hsi means siRNA against HECTD3. (e) HECTD3 depletion in MDA-MB-231 significantly decreased the colony formation in the presence of TRAIL. MDA-MB-231 cells were seeded in 12-well plates at 100/well. LusiRNA and HECTD3 siRNA were transfected on the second day. Two days later, the cells were left untreated or treated with $10 \mathrm{ng} / \mathrm{ml}$ TRAIL for 2 weeks. The quantitative results are shown on the right panel. Error bars are S.D. from three independent experiments. ${ }^{* *} P<0.01$; $t$-test 

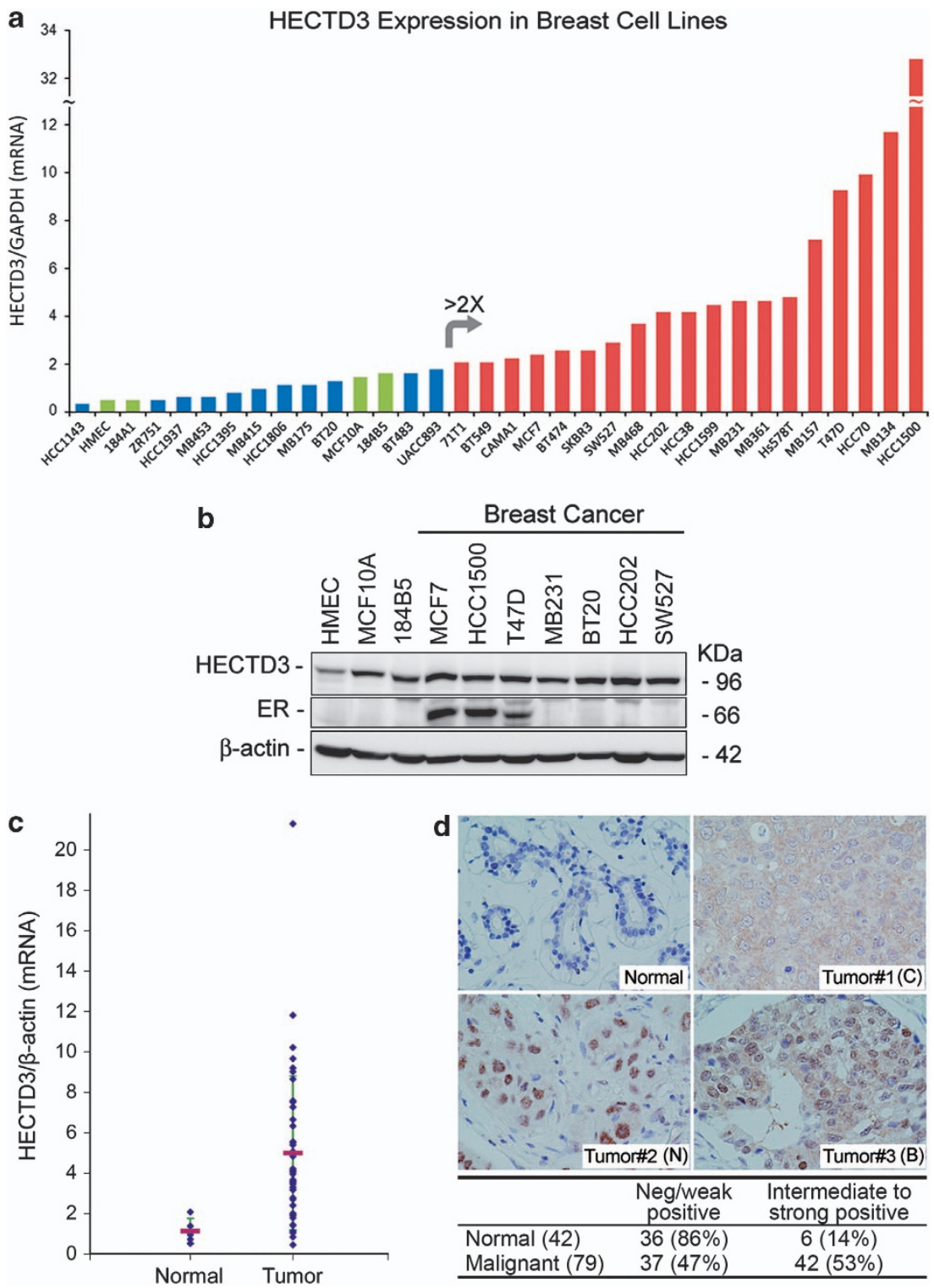

Figure 5 The HECTD3 expression is elevated in breast cancer. (a) The HECTD3 mRNA levels in 30 breast cancer cell lines and four control samples were measured by qRT-PCR. GAPDH was used to normalize the input CDNA. The negative control samples (the average HECTD3 mRNA level was defined as 1.0) are indicated as green bars. Cancer samples (19/30) with the HECTD3 overexpression (>2-fold) are highlighted in red. The cancer samples without the HECTD3 overexpression are represented as blue bars. MB in cell line names is an abbreviation of MDA-MB. (b) The expression of the HECTD3 protein in breast cell lines was determined by WB. $\beta$-Actin was used as the loading control. The ER $\alpha$ expression in these cell lines is also shown. (c) The HECTD3 mRNA levels in 48 breast tissues (5 normal and 43 cancer specimens) in different stages. The CDNA levels were normalized by $\beta$-actin. (d) HECTD3 protein expression in normal breast tissues and breast carcinomas specimens detected by IHC. Tumor\#1 demonstrates diffuse, weak cytoplasmic immunoreactivity (C, $25 \%$ of all tumors). Tumor\#2 demonstrates diffuse nuclear immunostaining ( $N, 42 \%$ of all tumors). Tumor\#3 demonstrates both cytoplasmic and nuclear immunostaining (B, $36 \%$ of all tumors). The IHC results from 42 normal and 79 breast cancer samples were summarized in the table below the image

levels, the HECTD3 protein levels were found to be low in the HMEC nontransformed breast cell line but elevated in breast cancer cell lines.

To confirm whether HECTD3 is also overexpressed in breast tumors, the HECTD3 mRNA levels were measured in a panel of breast tumors by qRT-PCR. The average HECTD 3 mRNA levels at normal breast and breast tumors are plotted in Figure 5c. Compared with normal breast tissues, breast tumors showed significantly elevated expression of the HECTD3 mRNA $(P<0.05, t$-test $)$. Furthermore, HECTD3 protein expression was also examined in normal breast tissues and breast carcinomas by $\mathrm{IHC}$ (Figure 5d). The anti-HECTD3 antibody was validated to specifically detect the HECTD3 protein by IHC (Supplementary Figure S5B). The majority $(86 \%, 36 / 42)$ of normal breast tissue sample and normal breast tissues adjacent to invasive carcinomas are negative for HECTD3 expression. In contrast, HECTD3 protein is overexpressed in 42 of $79(53 \%)$ of breast carcinomas $(P=0.0002$, Fisher's exact test) and localized in both the cytoplasm and the nucleus. 


\section{Discussion}

E3 ligases, such as Mdm2 and IAPs, have been well documented to inhibit apoptosis. The present data support a model that a novel HECT type E3 ligase HECTD3 inhibits the extrinsic apoptosis pathway at least partially through ubiquitinating caspase-8 with K63-linked polyubiquitin chains in human cancers. HECTD3 is frequently overexpressed in breast cancer cell lines and tumors at both mRNA and protein levels. HECTD3 depletion sensitized cancer cell lines to TRAIL, TNF $\alpha$, and Fas antibody induced apoptosis. Ectopic expression of HECTD3 conferred MDA-MB-231 TRAIL resistance in an E3 ligase activity-dependent manner. HECTD3 interacts with caspase-8, ubiquitinates caspase-8 with K63-linked polyubiquitin chains at K215, and reduces its recruitment into DISC for activation. Taken together, the HECTD3 E3 ligase overexpression in breast cancers promotes cancer cell survival from extrinsic apoptotic stimuli at least partially through ubiquitinating caspase-8 with K63-linked polyubiquitin chains (Supplementary Figure S7).

HECTD3 interacts with caspase-8 through the DOC-DED interactions (Figure 1). Several other E3 ubiquitin ligases, such as APC10, CUL7, PARC, and HERC2, contain a DOC domain. ${ }^{36}$ The DOC domain of APC10 contributes to substrate recognition for the anaphase-promoting complex (APC) E3 ligase, ${ }^{37}$ although the DOC-binding partner is unknown. Here, we demonstrated that the DOC domain of HECTD3 binds to the DED domain of caspase-8 (Figure 1) and DD domain of MALT1. ${ }^{29}$ It would be interesting to test whether other DOC domain-containing E3 ligases also interact with caspase-8. There are several other DED domain-containing proteins, such as FLIP, FADD, PEA-15, and DEDD. ${ }^{38}$ HECTD3 did not interact with FLIP . These results indicate that the interaction between HECTD3 and caspase- 8 is specific.

The data presented in this study indicate for the first time that HECTD3 promotes cell survival partially through ubiquitinating caspase-8 with K63-linked polyubiquitin chains at K215. Ubiquitination-mediated inactivation of caspases has been long linked to apoptosis resistance. ${ }^{39}$ Ditzel et al. ${ }^{40}$ reported that the DIAP1 E3 ligase inactivates effector caspase (drICE) through nondegradative polyubiquitination in Drosophila. The current study provides the first evidence that HECTD3-mediated caspase-8 K63-linked polyubiquitination suppresses the activation of caspase-8. Caspase- 8 is well known to be recruited into DISC for self-cleavage and activation. Depletion of HECTD3 increases the caspase-8 recruitment and activation. In contrast to the CUL3-mediated caspase-8 polyubiquitination at the C-terminus, HECTD3mediated caspase-8 polyubiquitination at K215 decreases its activation. This mechanism is also different from TRAF2mediated K48-linked polyubiquitination on the large catalytic domain of caspase- $8^{14}$ and TRIM13-mediated K63-linked caspase-8 polyubiquitination, which results in caspase-8 activation during ER stress. ${ }^{41}$

$\mathrm{Yu}$ and his colleagues previously reported that HECTD3 is the E3 ligase for Tara. ${ }^{27}$ An N-terminal-truncated HECTD3 (284-861) was shown to interact with exogenous Tara and to target exogenous Tara for ubiquitin-mediated proteasomal degradation. ${ }^{27}$ In another report, Zhang et al. ${ }^{28}$ identified the Syntaxin-8 protein as a HECTD3 interacting protein by yeast two-hybrid screening and demonstrated that HECTD3 increases the Syntaxin-8 ubiquitination in HEK293T cells. Syntaxin-8 is a vesicle trafficking protein associated with neurodegenerative diseases. ${ }^{42}$ Thus, it cannot be completely excluded the possibility that HECTD3 promotes cell survival partially by targeting Tara and/or Syntaxin-8 for degradation. Our previous study showed that HECTD3 stabilizes MALT1 and protects cancer cells from cisplatin-induced apoptosis. ${ }^{29}$ MALT1 and caspase-8 are known to form a protein complex. ${ }^{30}$ Thus, it seems that HECTD3 functions partially through both MALT1 and Caspase-8. It is also possible that HECTD3 possesses other unidentified functions by regulating Tara, Syntaxin-8, or other substrates.

HECTD3 is a pro-survival protein overexpressed in breast cancers. Whether the HECTD3 expression level is an ideal biomarker for breast cancer diagnosis and prognosis requires larger scale IHC studies in the future. The future HECTD3targeted therapy should combine with other therapies such as TRAIL and cisplatin.

\section{Materials and Methods}

Cell culture. MDA-MB-231 was cultured in DMEM supplemented with $2 \mathrm{mM}$ L-glutamine, $0.1 \mathrm{mM}$ non essential amino acid, $1.5 \mathrm{~g} / \mathrm{l}$ sodium bicarbonate, $1 \mathrm{mM}$ sodium pyruvate and 10\% FBS. HeLa was cultured in DMEM supplemented with $5 \% \mathrm{FBS}$ and $1 \% \mathrm{PS}$.

Antibodies. The anti-HECTD3 rabbit polyclonal antibody was described in our previous study. ${ }^{29}$ The anti-HECTD3 antibody was validated by the exogenous expressed HECTD3 protein by using a standard WB protocol (Supplementary Figure S5B). The anti- $\beta$-actin mouse monoclonal antibody, the anti-Flag rabbit polyclonal antibody, the anti-PARP, anti-caspase-7, anti-cl-caspase-7, anti-caspase-8, and anti-caspase-3 antibodies are from Cell Signaling (Danvers, MA, USA). The rabbit anti-caspase- 8 antibody is from R\&D systems. The anti-DR4 antibody is from DIACLONE (Tepnel Company, France). The anti-DR5 antibody is from ProSci Inc. (Poway, CA, USA). The anti-FADD, anti-Ub, anti-ER $\alpha$, and anti-HA antibodies are from Santa Cruz Biotechnology (Santa Cruz, CA, USA). The anti-GST antibody and anti-Flag M2 monoclonal antibody are from Sigma (St. Louis, MO, USA). Ubiquitin linkage-specific antibodies (Apu2.07 and Apu3.A8) are gifts from Dr. Dixit, V. M. in Genentech. ${ }^{31}$

Plasmids. The endogenous HECTD3 protein is about $96 \mathrm{KDa}$, which is consistent with the open reading frame of the HECTD3 mRNA sequence (NM_024602.5) (Supplementary Figure S8). The full-length HECTD3 gene was cloned into the pLenti6/V5-D vector as described. ${ }^{29}$ MDA-MB-231 cells were transduced with WT HECTD3 and mutant HECTD3-C774, 823A respectively. The cell populations were obtained after selection with $10 \mu \mathrm{g} / \mathrm{ml}$ blasticidin. The deletion mutants of Flag-HECTD3 $(\Delta 1-215, \Delta 109-393, \Delta 216-393, \Delta 394-511$, $\Delta 1-511$, and $\Delta 512-861)$ and HECTD3 mutants were generated by a PCRbased approach. To generate HECTD3 shRNA (Hsh)-resistant HECTD3 cDNA, we silently mutated 10 nucleotides within the shRNA target sequence (from 5'-GCGGGAACTAGGGTTGAAT-3' to $5^{\prime}$-AAGAGAGTTGGGTCTAAAC-3'). Different regions of HECTD3 were cloned into $\mathrm{DEBG}$ and pGEX-6p-1 vectors to produce GST-fusion proteins in mammalian cells and in E.coli, respectively. The pcDNA3-caspase-8 (NM_001228) plasmid is a gift from Dr. Gabriel Nuñez from University of Michigan. pEF-IRES-P-HA-Ub and Ub mutants (KO, K48-only, K63-only, K48R, and K63R) are gifts from Dr. James Zhijian Chen (University of Texas Southwestern Medical Center). ${ }^{43}$

Transfection. All transfections for plasmids and siRNAs were performed using Lipofectamine 2000 (Invitrogen) according to the manufacturer's instruction. All chemically synthesized siRNAs were purchased from Ambion (Austin, TX, USA) and transfected at $25 \mathrm{nM}$ final concentration. The siRNA target sequences for the human HECTD3 gene are 5'- GCGGGAACTAGGGTTGAAT-3'. The sequence was chosen for preparing the HECTD3 shRNA construct in the pSIH1-H1-Puro 
shRNA vector. The siRNA target sequence for the human caspase-8 gene is 5' - GATCAGAATTGAGGTCTTT- 3'.

Ubiquitination assays. HEK293T cells were transiently transfected with $\mathrm{HA}-\mathrm{Ub}$ and other plasmids as necessary in six-well plates. Two days after transfection, the cells were collected in $150 \mu \mathrm{l}$ SDS lysis buffer $(50 \mathrm{mM}$ Tris-Cl, $\mathrm{pH}$ $6.8,1.5 \% \mathrm{SDS}$ ). The samples were boiled for $15 \mathrm{~min}$. One hundred microliters of protein lysate was diluted with $1.2 \mathrm{ml}$ EBC/BSA buffer $(50 \mathrm{mM}$ Tris-Cl, $\mathrm{pH} 6.8$, $180 \mathrm{mM} \mathrm{NaCl}, 0.5 \%$ CA630, 0.5\% BSA) and incubated with anti-Flag M2-agarose overnight at $4{ }^{\circ} \mathrm{C}$ with rotation. The beads were collected by centrifugation at $10000 \times g$ for $30 \mathrm{~s}$ at $4^{\circ} \mathrm{C}$ and washed three times with $1 \mathrm{ml}$ ice-cold EBC/BSA buffer. Proteins were resuspended with $30 \mu \mathrm{l}$ of $3 \times$ SDS sample loading buffer and analyzed by WB. Ubiquitinated caspase- 8 was detected by an anti-HA antibody.

The HECTD3 self-ubiquitination assay was performed with recombinant-purified GST-HECTD3, GST-HECTD3-C823A, E1, E2 (UbcH5b), Ub, and ATP in a 15- $\mu$ l system. The reactions were incubated at $30^{\circ} \mathrm{C}$ for $3 \mathrm{~h}$. The ubiquitinated HECTD3 proteins were detected by WB using the anti-Ub antibody.

DISC formation. MDA-MB-231 cells ( $\sim 1 \times 10^{7}$ Lush and Hsh) were grown in a $15-\mathrm{cm}$ dish and collected in $2 \mathrm{ml}$ complete DMEM. The recombinant human soluble Flag-tagged TRAIL ( $1 \mu \mathrm{g}$, Alexis) and the anti-Flag M2 agarose $(4.6 \mu \mathrm{g})$ were premixed for $15 \mathrm{~min}$ on ice in a $1.5 \mathrm{ml}$ Eppendorf tube. The cells were added into the tube for $30 \mathrm{~min}$ at $37^{\circ} \mathrm{C}$ with gentle shaking. The reaction was stopped by the addition of $10 \mathrm{ml}$ ice-cold PBS. The cells were immediately collected (400 g, $5 \mathrm{~min}, 4^{\circ} \mathrm{C}$ ), washed with $1 \mathrm{ml}$ ice-cold PBS, and lysed in $1 \mathrm{ml}$ lysis buffer for $15 \mathrm{~min}$ on ice. The lysate was centrifuged twice at $16000 \mathrm{~g}$ for $10 \mathrm{~min}$ at $4{ }^{\circ} \mathrm{C}$. In unstimulated controls, $1 \mu \mathrm{g}$ Flag-TRAIL and the anti-Flag M2 agarose were directly added into the cell lysate. Beads were recovered by centrifugation, washed five times with $500 \mu$ l lysis buffer, and subjected to WB.

HECTD3 mRNA analysis. The HECTD3 mRNA levels were examined by qRT-PCR as described in our previous studies. ${ }^{44,45}$ Primer sequences for HECTD3 are 5'- GGGCTACGAGACCACAGACG $-3^{\prime}$ and 5'- GCAAGGCCAA GAGGGACAC - $3^{\prime}$. All qPCRs were performed in duplicate. GAPDH and $\beta$-actin were used as loading controls. HECTD3 overexpression is defined when the ratio in a cancer sample is larger than two compared with the average ratio in all normal samples. cDNA from 48 breast tissues (BCRT102) and normal tissues (HMRT102) was purchased from Origene (Rockville, MD, USA).

Immunohistochemical staining. To confirm the specificity of the antiHECTD3 antibody for IHC, the HEK293T cells (transfected with HECTD3 and an empty vector control) were stained by using an indirect biotin avidin diaminobenzidine detection system according to the routine $\mathrm{IHC}$ procedure (Supplementary Figure S5B). After validating the anti-HECTD3 antibody for IHC, normal breast and breast carcinoma tissue microarrays were purchased from US Biomax, Inc (BC08022 and BC08014). In total, 42 different normal or adjacent $(1.5 \mathrm{~cm}$ away from the tumor) normal breast tissues and 79 breast carcinomas were stained. Slides were baked at $60^{\circ} \mathrm{C}$ for $2 \mathrm{~h}$ and underwent antigen retrieval procedures before regular IHC. The anti-HECTD3 primary antibody was used at a dilution of 1:100. Immunoreactivity for HECTD3 was interpreted by two pathologists without previous knowledge of the clinicopathologic parameters. Immunostained slides were evaluated by light microscopy, and the IHC signal was scored using the 'Allred Score' method. ${ }^{46}$

Immunoprecipitation and GST pull-down. Immunoprecipitation using anti-Flag M2-agarose (Sigma, \#A2220) and GST pull-down experiments have been described in our previous studies. ${ }^{23}$ The anti-HECTD3 rabbit polyclonal antibody was used to immunoprecipitate the endogenous HECTD3 proteins from HeLa. The rabbit IgG was used as the negative control.

In vitro translation. Caspase- 8 and the caspase-3 proteins were synthesized from the pcDNA3-Caspase-8 or -3 plasmids using the TNT Quick Coupled Transcription/Translation Systems (Promega, Madison, WI, USA) in the presence of methionine, according to the manufacturer's protocol.

Apoptosis. Cells were transfected with the HECTD3 siRNA and control siRNA for 2 days. If applied, different concentrations of TRAIL were added for indicated time. The cell viability was measured by performing the SRB assay as described in our previous study. ${ }^{44}$ Cleaved PARP and cleaved caspase-3, $-7,-8$ protein levels were detected by WB. Annexin V/7-AAD (BD, Franklin Lakes, NJ, USA) staining was performed according to the manufacturer's protocol.

Statistical analysis. The cell viability experiments were conducted in triplicate and repeated at least once. The data were pooled to generate means \pm S.D. and analyzed by Student's $t$-test. The HECTD3 protein expression in tumors and the correlation between the HECTD3 gene copy-number gains and mRNA overexpression were analyzed by Fisher's exact test. $P$-values of $<0.05$ were considered to be significant.

\section{Conflict of Interest}

The authors declare no conflict of interest.

Acknowledgements. We thank Dr. Gabriel Nuñez from University of Michigan, Dr. James Zhijian Chen from University of Texas, Dr. Chunghong Yan from Albany Medical College, and Dr. Dixit, V. M. from Genentech for reagents. Drs. Gang Liu and Guoning Liao from Albany Medical College provided technical support. This study was supported in part by National Key Basic Research Program of China (No. 2013CB910900), grants from National Nature Science Foundation of China (81120108019, 81072162, and U1132605), and a grant from Yunnan Province High-Profile Scientist Program (2010Cl114).

1. Earnshaw WC, Martins LM, Kaufmann SH. Mammalian caspases: structure, activation, substrates, and functions during apoptosis. Annu Rev Biochem 1999; 68: 383-424.

2. Riedl SJ, Shi Y. Molecular mechanisms of caspase regulation during apoptosis. Nat Rev Mol Cell Biol 2004; 5: 897-907.

3. Boldin MP, Goncharov TM, Goltsev YV, Wallach D. Involvement of MACH, a novel MORT1/FADD-interacting protease, in Fas/APO-1- and TNF receptor-induced cell death. Cell 1996; 85: 803-815.

4. Chang DW, Xing Z, Capacio VL, Peter ME, Yang X. Interdimer processing mechanism of procaspase-8 activation. Embo J 2003; 22: 4132-4142.

5. Kurokawa M, Kornbluth S. Caspases and kinases in a death grip. Cell 2009; 138: 838-854.

6. Riedl SJ, Renatus M, Schwarzenbacher R, Zhou Q, Sun C, Fesik SW et al. Structural basis for the inhibition of caspase-3 by XIAP. Cell 2001; 104: 791-800.

7. Chai J, Shiozaki E, Srinivasula SM, Wu Q, Datta P, Alnemri ES et al. Structural basis of caspase-7 inhibition by XIAP. Cell 2001; 104: 769-780.

8. Shiozaki EN, Chai J, Rigotti DJ, Riedl SJ, Li P, Srinivasula SM et al. Mechanism of XIAP-mediated inhibition of caspase-9. Mol Cell 2003; 11: 519-527.

9. Huang $\mathrm{H}$, Joazeiro CA, Bonfoco E, Kamada S, Leverson JD, Hunter T. The inhibitor of apoptosis, cIAP2, functions as a ubiquitin-protein ligase and promotes in vitro monoubiquitination of caspases 3 and 7. J Biol Chem 2000; 275: 26661-26664.

10. Choi YE, Butterworth M, Malladi S, Duckett CS, Cohen GM, Bratton SB. The E3 ubiquitin ligase CIAP1 binds and ubiquitinates caspase- 3 and -7 via unique mechanisms at distinct steps in their processing. J Biol Chem 2009; 284: 12772-12782.

11. Jin Z, Li Y, Pitti R, Lawrence D, Pham VC, Lill JR et al. Cullin3-based polyubiquitination and p62-dependent aggregation of caspase-8 mediate extrinsic apoptosis signaling. Cell 2009; 137: 721-735.

12. Peng C, Cho YY, Zhu F, Zhang J, Wen W, Xu Y et al. Phosphorylation of caspase-8 (Thr-263) by ribosomal S6 kinase 2 (RSK2) mediates caspase-8 ubiquitination and stability. J Biol Chem 2011; 286: 6946-6954.

13. Christian PA, Fiandalo MV, Schwarze SR. Possible role of death receptor-mediated apoptosis by the E3 ubiquitin ligases Siah2 and POSH. Mol Cancer 2011; 10: 57.

14. Gonzalvez F, Lawrence D, Yang B, Yee S, Pitti R, Marsters S et al. TRAF2 Sets a threshold for extrinsic apoptosis by tagging caspase-8 with a ubiquitin shutoff timer. Mol Cell 2012; 48: 888-899.

15. Thorpe JA, Christian PA, Schwarze SR. Proteasome inhibition blocks caspase-8 degradation and sensitizes prostate cancer cells to death receptor-mediated apoptosis. Prostate 2008; 68: 200-209.

16. Fiandalo MV, Schwarze SR, Kyprianou N. Proteasomal regulation of caspase-8 in cancer cell apoptosis. Apoptosis 2013; 18: 766-776.

17. Broemer M, Meier P. Ubiquitin-mediated regulation of apoptosis. Trends Cell Biol 2009; 19: $130-140$.

18. McManus DC, Lefebvre CA, Cherton-Horvat G, St-Jean M, Kandimalla ER, Agrawal $S$ et al. Loss of XIAP protein expression by RNAi and antisense approaches sensitizes cancer cells to functionally diverse chemotherapeutics. Oncogene 2004; 23: 8105-8117.

19. Varfolomeev E, Blankenship JW, Wayson SM, Fedorova AV, Kayagaki N, Garg P et al. IAP antagonists induce autoubiquitination of c-IAPs, NF-kappaB activation, and TNFalpha-dependent apoptosis. Cell 2007; 131: 669-681. 
20. Bernassola F, Karin M, Ciechanover A, Melino G. The HECT family of E3 ubiquitin ligases: multiple players in cancer development. Cancer Cell 2008; 14: 10-21.

21. Garcia-Gonzalo FR, Rosa JL. The HERC proteins: functional and evolutionary insights Cell Mol Life Sci 2005; 62: 1826-1838.

22. Chen C, Matesic LE. The Nedd4-like family of E3 ubiquitin ligases and cancer. Cance Metastasis Rev 2007; 26: 587-604.

23. Li Y, Zhou Z, Chen C. WW domain-containing E3 ubiquitin protein ligase 1 targets p63 transcription factor for ubiquitin-mediated proteasomal degradation and regulates apoptosis. Cell Death Differ 2008; 15: 1941-1951.

24. Zhong Q, Gao W, Du F, Wang X. Mule/ARF-BP1, a BH3-only E3 ubiquitin ligase, catalyzes the polyubiquitination of Mcl-1 and regulates apoptosis. Cell 2005; 121: 1085-1095.

25. Pal M, Varga K, Nagy O, Deak P. Characterization of the Apc10/Doc1 subunit of the anaphase promoting complex in Drosophila melanogaster. Acta Biol Hung 2007; 58(Suppl): 51-64.

26. Kaustov L, Lukin J, Lemak A, Duan S, Ho M, Doherty $R$ et al. The conserved CPH domains of Cul7 and PARC are protein-protein interaction modules that bind the tetramerization domain of p53. J Biol Chem 2007; 282: 11300-11307.

27. Yu J, Lan J, Zhu Y, Li X, Lai X, Xue Y et al. The E3 ubiquitin ligase HECTD3 regulates ubiquitination and degradation of Tara. Biochem Biophys Res Commun 2008; 367 805-812.

28. Zhang L, Kang L, Bond W, Zhang N. Interaction between syntaxin 8 and HECTd3, a HECT domain ligase. Cell Mol Neurobiol 2009; 29: 115-121.

29. Li Y, Chen X, Wang Z, Zhao D, Chen H, Chen W et al. The HECTD3 E3 Ubiquitin Ligase Suppresses Cisplatin-Induced Apoptosis via Stabilizing MALT1. Neoplasia 2013; 15: 39-48.

30. Kawadler H, Gantz MA, Riley JL, Yang X. The paracaspase MALT1 controls caspase-8 activation during lymphocyte proliferation. Mol Cell 2008; 31: 415-421.

31. Newton K, Matsumoto ML, Wertz IE, Kirkpatrick DS, Lill JR, Tan J et al. Ubiquitin chain editing revealed by polyubiquitin linkage-specific antibodies. Cell 2008; 134: 668-678.

32. Huang L, Kinnucan E, Wang G, Beaudenon S, Howley PM, Huibregtse JM et al. Structure of an E6AP-UbcH7 complex: insights into ubiquitination by the E2-E3 enzyme cascade. Science 1999; 286: 1321-1326.

33. Medema JP, Scaffidi C, Krammer PH, Peter ME. Bcl-xL acts downstream of caspase-8 activation by the CD95 death-inducing signaling complex. J Biol Chem 1998; 273: 3388-3393.

34. Oberst A, Pop C, Tremblay AG, Blais V, Denault JB, Salvesen GS et al. Inducible dimerization and inducible cleavage reveal a requirement for both processes in caspase-8 activation. J Biol Chem 2010; 285: 16632-16642.

35. Cohen GM. Caspases: the executioners of apoptosis. Biochem J 1997; 326(Pt 1): 1-16.
36. Dias DC, Dolios G, Wang $\mathrm{R}$, Pan ZQ CUL7: A DOC domain-containing cullin selectively binds Skp1.Fbx29 to form an SCF-like complex. Proc Natl Acad Sci USA 2002; 99: 16601-16606.

37. Passmore LA, McCormack EA, Au SW, Paul A, Willison KR, Harper JW et al. Doc1 mediates the activity of the anaphase-promoting complex by contributing to substrate recognition. Embo J 2003; 22: 786-796.

38. Valmiki MG, Ramos JW. Death effector domain-containing proteins. Cell Mol Life Sci 2009; 66: $814-830$

39. Mace PD, Shirley S, Day CL. Assembling the building blocks: structure and function of inhibitor of apoptosis proteins. Cell Death Differ 2010; 17: 46-53.

40. Ditzel M, Broemer M, Tenev T, Bolduc C, Lee TV, Rigbolt KT et al. Inactivation of effector caspases through nondegradative polyubiquitylation. Mol Cell 2008; 32: 540-553.

41. Tomar D, Prajapati $\mathrm{P}$, Sripada L, Singh K, Singh R, Singh AK et al. TRIM13 regulates caspase-8 ubiquitination, translocation to autophagosomes and activation during ER stress induced cell death. Biochim Biophys Acta 2013; 1833: 3134-3144.

42. Thoreau V, Berges T, Callebaut I, Guillier-Gencik Z, Gressin L, Bernheim A et al. Molecular cloning, expression analysis, and chromosomal localization of human syntaxin 8 (STX8). Biochem Biophys Res Commun 1999; 257: 577-583.

43. Zeng W, Xu M, Liu S, Sun L, Chen ZJ. Key role of Ubc5 and lysine-63 polyubiquitination in viral activation of IRF3. Mol Cell 2009; 36: 315-325.

44. Chen C, Zhou Z, Ross JS, Zhou W, Dong JT. The amplified WWP1 gene is a potential molecular target in breast cancer. Int J Cancer 2007; 121: 2834-2841.

45. Chen $C$, Sun $X$, Guo $P$, Dong $X Y$, Sethi $P$, Zhou $W$ et al. Ubiquitin E3 ligase WWP1 as an oncogenic factor in human prostate cancer. Oncogene 2007; 26: 2386-2394

46. Mohsin SK, Weiss H, Havighurst T, Clark GM, Berardo M, Roanh le D et al. Progesterone receptor by immunohistochemistry and clinical outcome in breast cancer: a validation study. Mod Pathol 2004; 17: 1545-1554.

47. Larkin MA, Blackshields G, Brown NP, Chenna R, McGettigan PA, McWilliam $\mathrm{H}$ et al. Clustal W and Clustal X version 2.0. Bioinformatics 2007; 23: 2947-2948.

(c) (i) $(9)$ Cell Death and Disease is an open-access journal published by Nature Publishing Group. This work is licensed under a Creative Commons Attribution-NonCommercialNoDerivs 3.0 Unported License. To view a copy of this license, visit http://creativecommons.org/licenses/by-nc-nd/3.0/

\section{Supplementary Information accompanies this paper on Cell Death and Disease website (http://www.nature.com/cddis)}

